\title{
Operational transfer path analysis method using several measurement systems
}

\author{
Junji YOSHIDA*, Takashi YAMAMOTO* and Yuta NOZUCHI* \\ *Osaka Institute of Technology \\ 5-16-1 Omiya, Asahi-Ku, Osaka 535-8585, Japan \\ E-mail: junji.yoshida@oit.ac.jp
}

Received: 29 April 2021; Revised: 11 July 2021; Accepted: 29 July 2021

\begin{abstract}
In this study, we developed a method for obtaining high contributing part (reference point) to the response point at the operational condition by operational TPA (OTPA) using several measurement systems. OTPA calculates contribution of each reference point to the response point using only operational signals. All reference and response signals are necessary to be measured simultaneously by single measurement system because the method calculates the contribution using their correlation along time. However, this occasionally requires preparing large measurement system depending on the number of measurement points and the size of products. This may decrease the applicability of the method. We then considered a post processing procedure to obtain accurate contribution of each reference point to the response point by using several measurement systems instead of preparing large single measurement system. In the proposed method, all signals are measured using different several systems at around same timing. The exact sampling timing gaps among systems are estimated by using the estimation error between the calculated and actual measured response signal. After then, all reference signals compensated by the estimated time gap in each system are regarded to be measured simultaneously and contribution of all reference signals are calculated by OTPA. As the verification of the proposed method, the procedure was applied to a simple vehicle model. As the result, the contribution of the proposed method was similar with the contribution by single measurement system and clarified to have an ability to obtain correct contribution by several measurement systems.
\end{abstract}

Keywords : Operational transfer path analysis, Time gap, Interior noise, Measurement system, Estimation error

\section{Introduction}

For carrying out effective countermeasure to product noise and vibration, finding out the main contributor among many parts is essential. Transfer path analysis (TPA) has been proposed to obtain the contribution quantitatively and several methods were developed until now (Plunt, 1998; Noumura, 2006; Brandl, 2008; Lohrmann, 2008; Klerk, D., 2010; Putner, 2012). Operational TPA (OTPA) is one of the methods recently developed and enable us to obtain the contribution of each part (reference point) to the response point with small man-hour because the method calculates the contribution using only the sound and vibration signals at the operational condition (Noumura, 2006; Lohrmann, 2008; Klerk, D., 2010; Putner, 2012; Yoshida, 2016; Yoshida, 2017; Hirao, 2019). For applying the method, all reference and response point signals are necessary to be measured simultaneously since the method calculates the contribution from the relationship between reference and the response signals along time. Thus, if we have a measurement system which can record a lot of signals, OTPA can be applied. On the other hand, in case the number of the reference point is very large (e.g., over 100 points), applying OTPA becomes hard according to the upper limit of the employed system. In this situation, using several measurement systems linking each other by external cable is one of the solution. However, if the target product or structure is very large (e.g., over $100 \mathrm{~m}$ ), using very long external cable for the connection is time and cost consuming and this may deteriorate the accuracy of OTPA. In a previous study, OTPA accuracy using several measurement systems linked by optical cable or GPS were actually investigated (Nozuchi, 2020). The result indicates that GPS link was not enough to obtain accurate contribution. In case of the optical cable link, the contribution accuracy 
was better than the GPS linked system. However, we have to prepare special measurement systems equipped with optical cable connection. This also decrease applicability of OTPA. In this study, we then considered a post processing procedure to obtain accurate contribution of each reference point to the response point using several measurement systems without any special additional link system.

\section{Contribution calculation by OTPA using several measurement systems 2.1. OTPA calculation flow}

In the original OTPA, the contribution of each reference point to the response point is obtained by multiplying each reference signal with the transfer function. The transfer function in this method is calculated using principal component regression method. The calculation procedure is shown in Fig. 1 (Noumura, 2006).

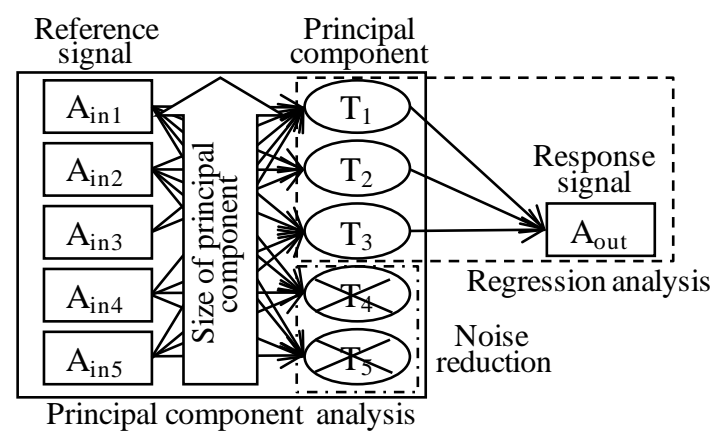

Fig. 1 Calculation image of transfer function of original OTPA using principal component regression method. Left solid box part indicates principal component analysis. Reference signals $\left(A_{\text {in }}\right)$ are transposed to principal components $(T)$ by the principal component analysis using singular value decomposition. Right dotted box part indicates regression analysis. The influence of each principal component $(T)$ on the response signal $\left(A_{\text {out }}\right)$ is obtained in this part.

The calculation procedure of OTPA is described as follows.

Firstly, PC analysis is applied to the reference signal matrix $\left[A_{\text {in }}\right]$ by singular value decomposition (SVD) to remove correlation among reference signals as shown in Eqs. (1) and (2). The calculated uncorrelated signals are PC [T].

$$
\begin{aligned}
& {\left[A_{\text {in }}\right]=[U][S \llbracket V]^{T}} \\
& {[T]=\left[A_{\text {in }}\right][V]=[U][S]}
\end{aligned}
$$

The reference signal matrix $\left[A_{\text {in }}\right]$ is obtained by applying FFT repeatedly to the simultaneously measured vibration signals at the reference points. The $(i, j)$-th element in the reference matrix $\left[A_{\text {in }}\right]$ is the data at the $j$-th reference point in the $i$-th FFT. Matrices $[U],[S]$, and $[V]$ are obtained as the result of SVD. Here, $[V]$ is the coefficient matrix to transpose the reference matrix $\left[A_{\text {in }}\right]$ to the principal component matrix $[T]$. The $(i, k)$-th element in $[T]$ is the $k$-th principal component in the $i$-th FFT.

After eliminating the noise component having very low level, multiple regression analysis is applied between the remained (signal) PCs $[T]$ and the response signal $\left[A_{\text {out }}\right]$ to obtain the influence $[B]$ of each principal component to the response signal as shown in Eqs. (3) and (4). Here, PC matrix [T] is not square matrix. Accordingly, squared matrix was made by multiplying transpose matrix $[T]^{T}$ and inverse matrix $\left([T]^{T}[T]^{T}\right)^{-1}$ was multiplied to obtain the influence matrix $[B]$ in Eq. (4).

$$
\begin{aligned}
& {\left[A_{\text {out }}\right]=[T][B]} \\
& {[B]=\left([T]^{T}[T]\right)^{-1}[T]^{T}\left[A_{\text {out }}\right]}
\end{aligned}
$$

The $(i, m)$-th element in the response matrix $\left[A_{\text {out }}\right]$ is the data at the $m$-th response point in the $i$-th FFT. The $(k, m)$-th element in the transfer matrix $[B]$ is the transpose coefficient from the $k$-th principal component to the $m$-th response point. 
Transfer function from reference signal to response signal $[H]$ is calculated by multiplying the coefficient $[V]$, that connects reference signal to principal component, and the regression coefficient $[B]$, that connects principal component and response signal as shown in Eq. (5)

$$
[H]=[V]\left([T]^{T}[T]\right)^{-1}[T]^{T}\left[A_{\text {out }}\right]
$$

The $(j, m)$-th element in the transfer matrix $[H]$ is the transfer function from the $j$-th reference point to the $m$-th response point.

Finally, the reference point contribution to the response point are calculated as shown in Eq. (6).

$$
\left[A_{\text {cont }}\right]=\left[A_{\text {in }}\right][H]
$$

Equation (6) shows the contribution of reference point. By multiplying each reference point signal $\left[A_{\text {in }}\right]$ with the transfer function $[H]$, reference point contribution $\left[A_{\text {cont }}\right]$ can be obtained. As described above, OTPA method calculates the contribution using the relationship between correlation between reference point and response point signals along time.

\subsection{Time gap compensation procedure among measurement systems}

Figure 2 shows the outline for estimating contributions of all reference point to the response point signals recorded by several measurement systems.

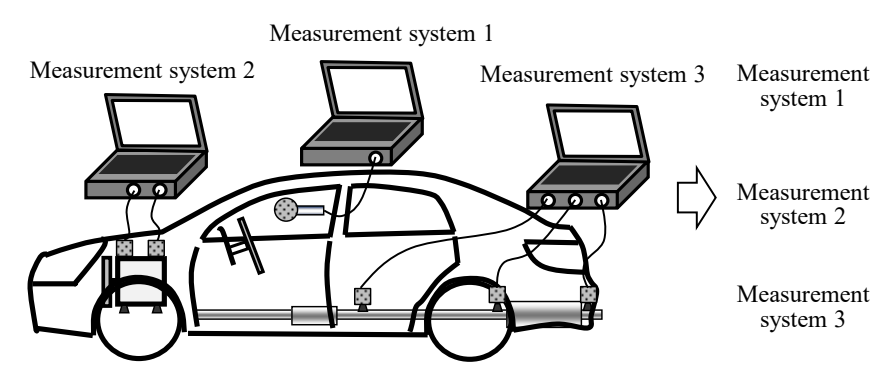

(a) Measurement using several systems.

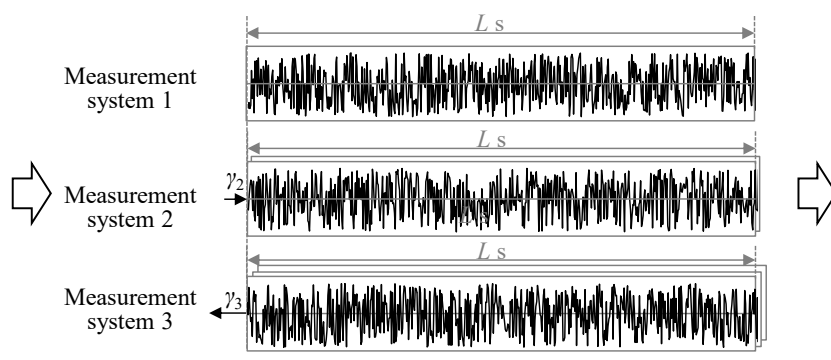

(c) Time gap compensated signals.

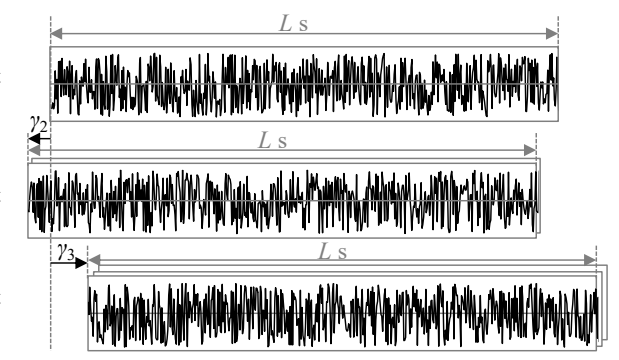

(b) Recorded signals including time gaps.

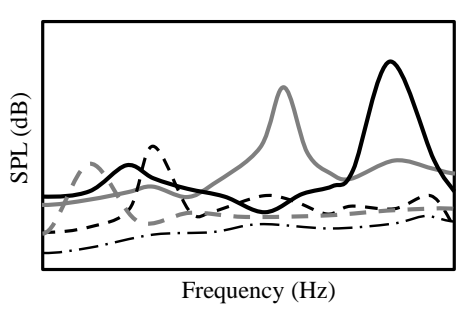

(d) Contribution by OTPA to compensated signals.

Fig. 2 Outline for estimating contribution by using several measurement systems. The reference and response signals are recorded using several systems at almost same timing as shown in (a). There is small time gap among the recorded signals as shown in (b). The time gap was estimated and the reference and response signals with the time gap are regards as signals measured simultaneously and OTPA is applied to the signals as shown in (c). The contribution of each part is calculated as if all signals were recorded by single measurement system as shown in (d).

As the first step, we separate the measurement group according to the number of the reference point and the position of reference and response point (Fig. 2 (a)). After then, all signals are recorded at around the same timing by several measurement systems. Here, the sampling timing of each system is not need to be exact same (Fig. 2 (b)). The sampling timing gap among them are compensated as follows in the third step (Fig. 2 (c)). After the compensation, all reference signals with the compensated time gap are regarded to be measured simultaneously and contribution of all reference signals are obtained by applying OTPA (Fig. 2 (d)).

For estimating the sampling timing gap among measurement systems, we attempted to utilize contribution error (SPL difference) obtained by OTPA. In OTPA method, transfer function from each reference point to the response is calculated to minimize the error between the summarized contribution from all reference points and the actual measured response 
signal. In other words, accurate transfer function can be obtained when the summarized contribution becomes closest to the actual measured response signal. As an instance, we discuss the process in case two measurement systems are employed as shown in Fig. 3. In this measurement, a measurement system is used to record only response signal and another system is prepared to record a reference signal and both systems are operated at around $L$ s. Here, there is a slight sampling timing gap $\gamma \mathrm{s}$ among them ( $\gamma \mathrm{s}$ delay in response measurement). In this condition, the error (deviation from calculated contribution from reference signal to actual measured response signal) is considered to be large as shown in right side of Fig. 3 (a). On the other hand, if the gap $\gamma$ is zero actually, the error is expected to be smallest as shown in right side of Fig. 3 (b). And in case the reference measurement is prior $\gamma \mathrm{s}$ to the response, the error also becomes large as shown in Fig. 3 (c).
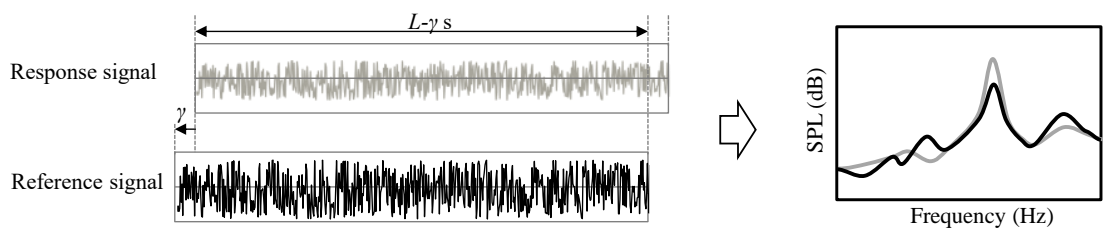

(a) Case1: Reference priors to response signal.

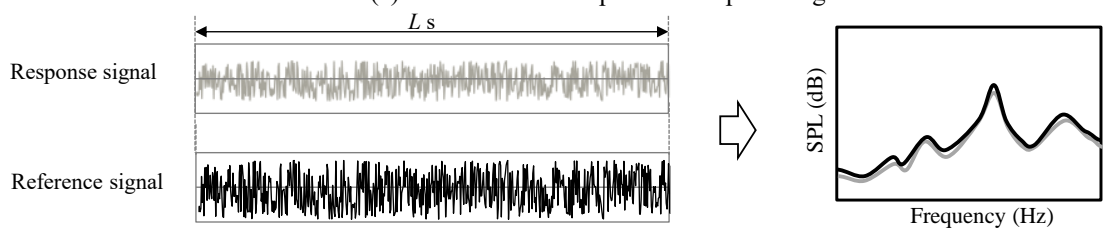

(b) Case2: Response and reference signals are recorded simultaneously.

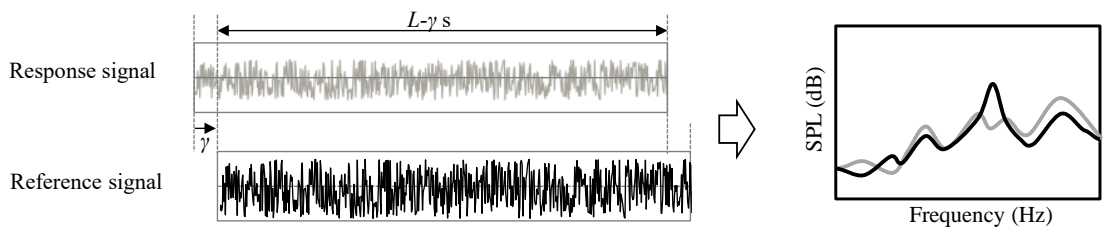

(c) Case3: Reference signal delays response signal.

Fig. 3 Relationship between measurement timing difference in two measurement system and the estimation error by OTPA. Reference point signal is prior to the response signal and the estimation error becomes large as shown in (a). In case the reference and response signals are recorded exactly same timing, the error is expected to be smallest as shown in (b). When the reference point signal delays slightly after response signal, the error also becomes large as shown in (c).

Then, we apply OTPA repeatedly at various time gap and calculate the contribution error in each time gap $(\gamma)$ to obtain relationship between the error and the time gap. When the error is the smallest, the time gap $(\gamma)$ is regarded to be the appropriate time gap for compensation among the measurement systems. This procedure is repeated in each reference point group and response point pair to obtain appropriate time gap $\gamma$ of each pair. Finally, OTPA is applied to all reference signals compensated by the estimated time gap in each group and the response signals. Through these processes, the contribution from all reference point to response point can be obtained using several measurement systems without using additional link equipment.

\section{Operational test and contribution calculation 3.1. Employed vehicle model and operational test}

Simple vehicle body model was prepared for the operational test as shown in Fig. 4. The length, width and height were $850 \times 300 \times 350 \mathrm{~mm}$. Total weight was $20 \mathrm{~kg}$ including four tires. Thickness of each body panel was $3 \mathrm{~mm}$ and the material of the panel was Aluminum. The cavity surrounding by the panel was regarded as the vehicle interior. 


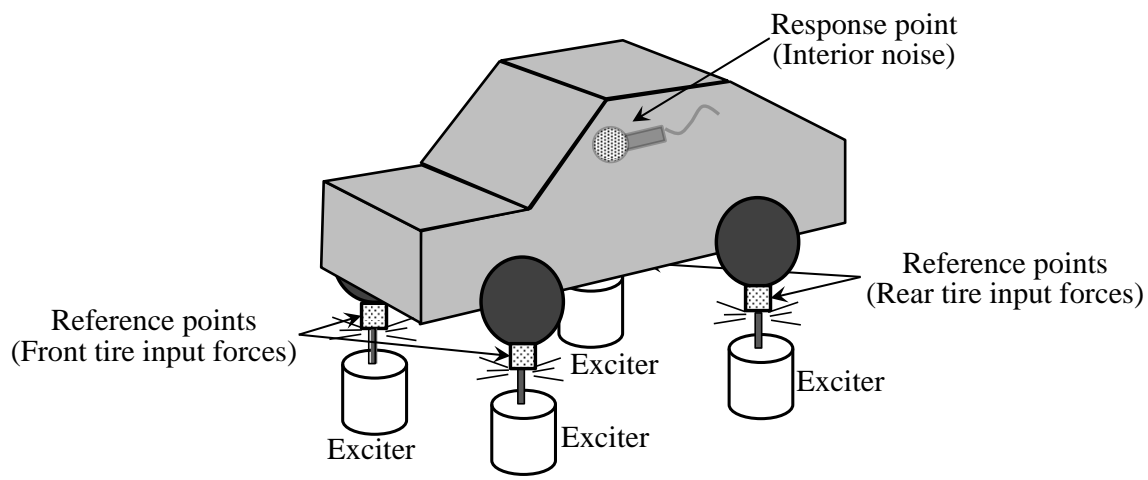

Fig. 4 Vehicle body physical model employed in the operational test. Input force was given from under the tire and interior noise was recorded as the response point signal. Input force to each tire was measured using force sensor as the reference point. As the response point, microphone was set in cabin to record interior noise.

As the input force, four electrical magnetic exciters (Modalshop: K2007E01) were put under four tires to give the forces. Uncorrelated random noise was given in each tire for about $20 \mathrm{~s}$. As the response point signal, vehicle interior noise was recorded by a microphone (PCB: 130E20) in cabin. As the reference point, four input force signals from exciter to each tire measured by force sensor (PCB: 208C02) was employed. Figure 5 shows the averaged SPL of interior noise at this operational condition.

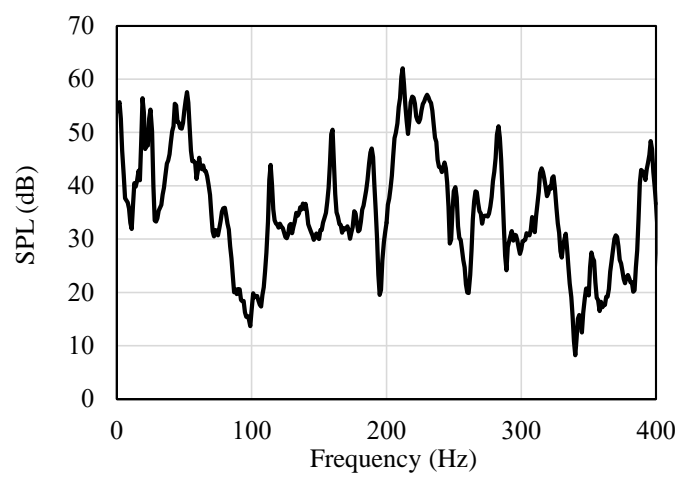

Fig. 5 Sound pressure level of the measured interior noise at the operational test. Horizontal and vertical axes show the frequency and the SPL, respectively.

In this operational test, we measured the reference and response signals at the following two patterns to verify the proposed method. In the first measurement pattern, all reference signals (four input force signals) and the response signal (interior noise) were recorded simultaneously by using single measurement system (Mueller-BBM: PAK system). In the second test pattern, input force signals to the two (left and right) front tires were recorded in a system (Mueller-BBM: PAK system), and input force signals to the two (left and right) rear tires were recorded by another system (MuellerBBM: PAK system). In addition, the response interior noise was recorded by the other system (HEAD acoustics: SQobold). This means three measurement systems were used in the second experimental pattern. The measurement by these three systems were started and finished at almost same timing for about $20 \mathrm{~s}$ as shown in Fig. 6. 


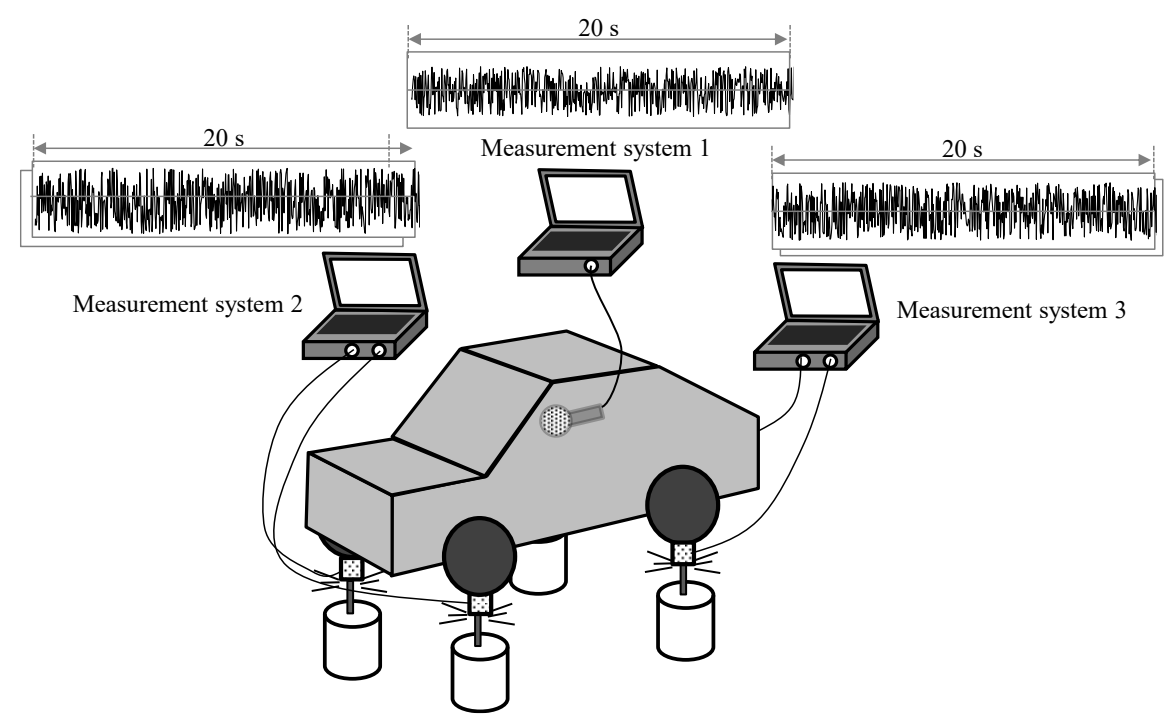

Fig. 6 Image of the measurement timing using three measurement systems in the second test pattern. Input force signals to the front tires were recorded in a measurement system, and input force signals to the rear tires were recorded by another system. In addition, the response interior noise was recorded by the other system. The measurement by these three systems were started and finished at almost same timing for about $20 \mathrm{~s}$

Original OTPA in the first pattern (single measurement system) was applied at first and the contributions of front and rear tires to the interior noise was obtained. And then, the accuracy of the proposed method in the second experimental pattern was verified by comparing the obtained contributions.

\subsection{Application of original OTPA by single measurement system}

As the first analysis, original OTPA by single measurement system was applied to the simultaneously recorded reference and response signals. Figure 7 shows the averaged input force contributions of front and rear tires. For obtaining front tire contributions the front-left and front-right contributions were summarized. Rear-left and rear-right contributions were also summarized for obtaining the rear tire contribution.

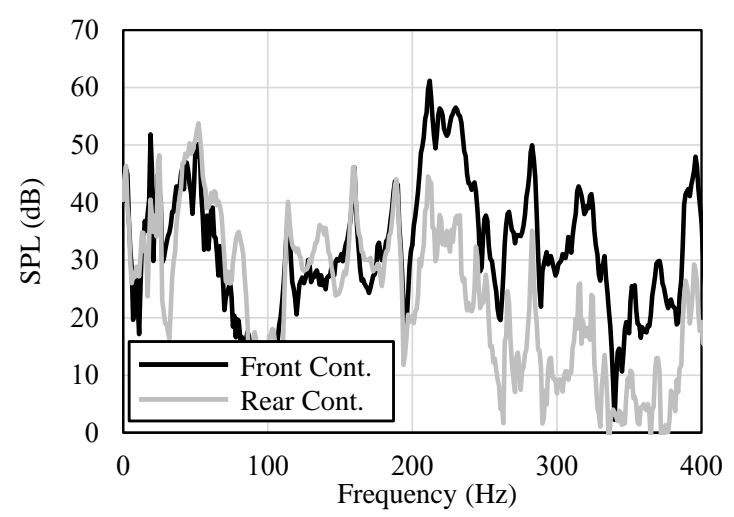

Fig. 7 Calculated front and rear tire contributions by original OTPA to the simultaneous recorded signals by single measurement system. Horizontal and vertical axes show the frequency and SPL, respectively. Black and gray curves show the front and rear contributions, respectively.

As shown in the upper figure, the front tire contribution was observed to be much larger than the rear tire contribution over $200 \mathrm{~Hz}$. In addition, the contribution peak at around $200 \mathrm{~Hz}$ was found to be made by the resonance between the body vibration mode of left and right side panel and the front input force by vibration mode analysis. This illustrates that if front tire input is decreased, the interior noise at this frequency band is expected to decrease. 
Then, for the verification of the original OTPA analytical result, we actually decreased the input forces to the front tires at around $200 \mathrm{~Hz}$ band and from $300 \mathrm{~Hz}$ to $400 \mathrm{~Hz}$ band, and recorded the interior noise again. Figure 8 shows the comparison of SPL before and after the front input force reduction.

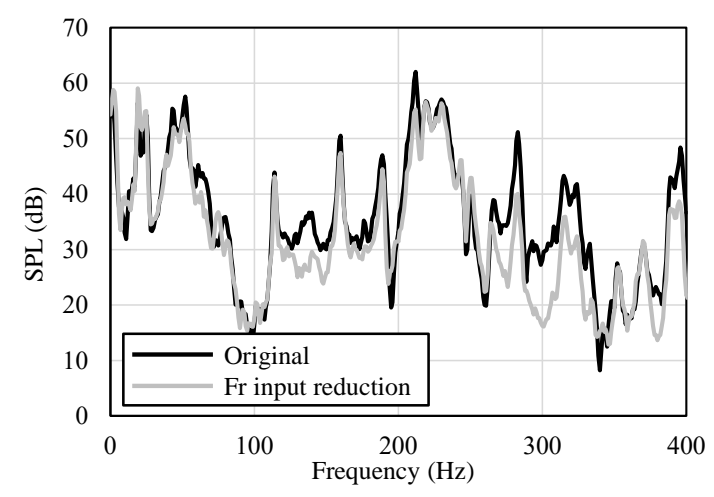

Fig. 8 SPL comparison before and after front input force reduction. Horizontal and vertical axes show the frequency and SPL, respectively. Black and gray curves show the interior noise before and after front input force reduction. The input force to the rear tires were constant in both conditions.

As shown in the Fig. 8, the interior noise was found to decrease at $200 \mathrm{~Hz}$ band and from $300 \mathrm{~Hz}$ to $400 \mathrm{~Hz}$ band, where the input force to the front tires were decreased. This indicates that original OTPA by single measurement system calculated correct contribution in this case. In the following section, we apply the proposed OTPA method using several measurement systems and compare the front and rear tire contributions with the original OTPA by single measurement system.

\subsection{Application of time gap compensation method for OTPA by several measurement systems}

For estimating appropriate time gap between the front tire reference point group and the response point (interior noise), we applied OTPA between the front tire input signals and the response signal recorded by using two measurement systems at various time gap $\left(\gamma_{\mathrm{f}}\right)$ and compared the summarized contribution (front tire contribution) with the response point. In this analysis, the time gap $\left(\gamma_{\mathrm{f}}\right)$ was set from $-2000 \mathrm{~ms}$ to $2000 \mathrm{~ms}$ at each $25 \mathrm{~ms}$ step considering the actual measurement start and stop timing difference. Subsequently, the SPL difference between the actual measured interior noise and calculated front tire contribution in each frequency was averaged for the target frequency range from 0 to $400 \mathrm{~Hz}$ at each time gap. Figure 9 (a) shows the relationship between the time gap $\gamma_{\mathrm{f}}$ and the averaged error. Figure 9 (b) also shows the relationship between the SPL difference and the time gap for rear tire signals $\left(\gamma_{\mathrm{r}}\right)$ in case OTPA was applied between rear tire input forces and the interior noise.

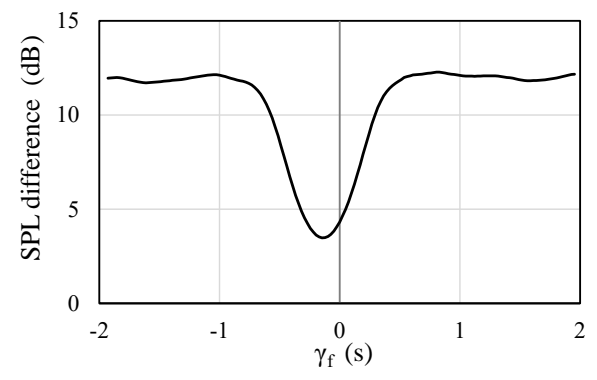

(a) Front tire measurement.

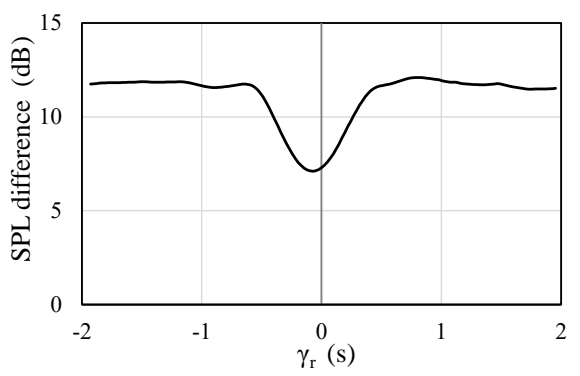

(b) Rear tire measurement.

Fig. 9 Relationship between the time gap and the averaged SPL difference. (a) shows the relationship in case the reference points were set at front tires and (b) shows the relationship in case the reference points were set at rear tires. Horizontal and vertical axes in both figures show the time gap and the averaged SPL difference.

As shown in Fig. 9 (a), apparent characteristic was observed when the gap $\left(\gamma_{\mathrm{f}}\right)$ was set at -150 ms. This illustrates that the front tire reference point measurement timing was prior $150 \mathrm{~ms}$ to the response point measurement. Hence, these reference point signals (front-left and front-right signals) can be regarded to be measured simultaneously with the response signal by delaying $150 \mathrm{~ms}$ on the reference signals of front tires. In case the analysis to rear tire signals, the SPL difference was smallest when the gap $\left(\gamma_{\mathrm{r}}\right)$ was set at $-50 \mathrm{~ms}$. Accordingly, these rear tire input signals seems to be 
measured $50 \mathrm{~ms}$ prior to the response signal measurement.

From these analytical results, we obtained the appropriate time gap in each measurement system for regarding all reference signals were measured simultaneously with the response signal. Then, the contributions of front and rear tire were calculated by applying OTPA to the time gap compensated reference signals and the response signal. Here, the front-left and -right tire input force signals were delayed $150 \mathrm{~ms}$ and rear-left and -right tire input force signals were delayed $50 \mathrm{~ms}$ for the compensation. Figure 10 shows the obtained front and rear tire contributions.

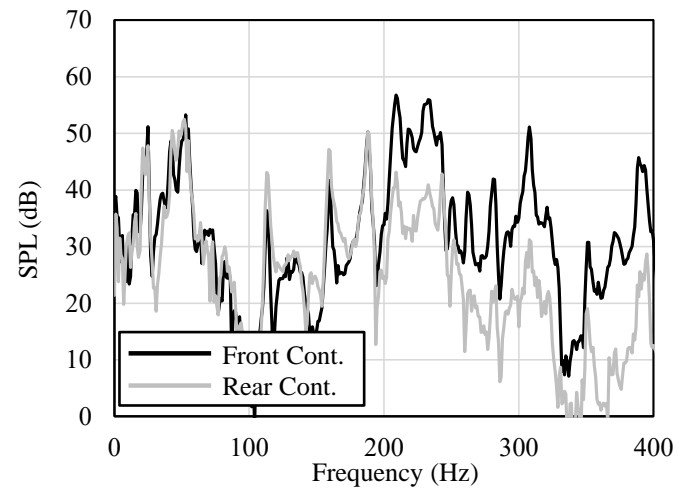

Fig. 10 Calculated front and rear tire contributions by OTPA to the gap compensated signals measured by several measurement systems. Horizontal and vertical axes show the frequency and SPL, respectively. Black and gray curves show the front and rear contributions, respectively.

As shown in Fig. 10, the front tire contribution was much larger than rear tire contribution over $200 \mathrm{~Hz}$. This tendency was the same as the contributions obtained by original OTPA using only single measurement systems as shown in Fig. 7. This indicates that the proposed method could calculate the accurate contribution by applying the proposed time gap compensation method to the reference signals measured by several measurement systems.

Here, we obtained front and rear tire contributions again in case inappropriate time gap was set at each front and rear input force signals as the other verification of the proposed method. From the relationship between the time gap and the SPL difference as shown in Fig. 9, we set +500 ms time gap for both front and rear signals as the inappropriate time gap, where the SPL difference was not small. And contribution from each reference signals with the time gap to the response point was obtained. Figure 11 shows the contribution of front and rear tire to the response point at this condition.

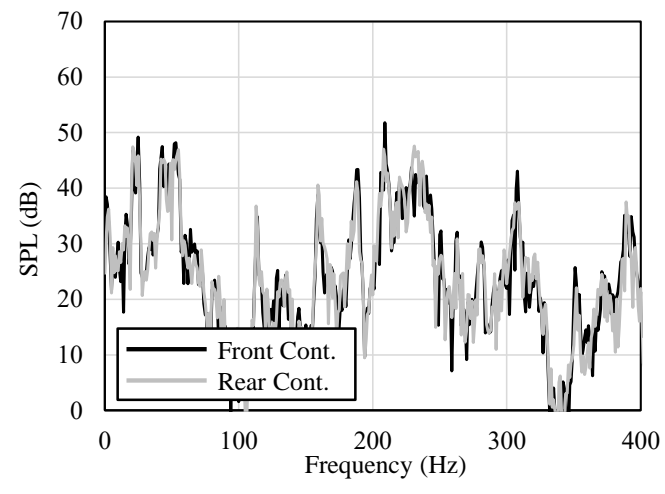

Fig. 11 Calculated front and rear tire contributions by applying OTPA to the inappropriate time $(+500 \mathrm{~ms})$ shifted reference signals and the response signal. Horizontal and vertical axes show the frequency and SPL, respectively. Black and gray curves show the front and rear contributions, respectively.

As shown in the upper figure, the obtained contribution was apparently different from the actual contribution by single measurement system (Fig. 7) and this was also quite different from the contribution by OTPA to the time gap compensated reference signals (Fig. 10). This also illustrates that the proposed time gap compensation method worked out well to obtain correct contributions by using several measurement systems. 


\section{Summary}

In this study, we proposed a method for obtaining high contributing part (reference point) to the response point at the operational condition by OTPA using several measurement systems. We then considered a post processing procedure to obtain accurate contribution. In this method, even though the response and reference point signals are measured by different measurement systems at almost same timing, the exact time gap among measurement systems are compensated by using the SPL difference between the estimated response signal and actual measured response signal. After obtaining the compensation time gap, all reference signals with the compensated time gap are regarded to be measured simultaneously and contribution of all reference signals are obtained as same as the original OTPA. As the verification of the proposal method, we actually applied this method to a simple vehicle model. In the test, input forces from four times (front-left, right, and rear-left right tires) were employed as the reference signals and interior noise was used as the response signal. The input forces to the front two tires and the forces to the rear two tires and interior noise were recorded using difference three measurement systems. Appropriate time gap for front tire input force signals and the gap for rear tire signals were estimated by evaluating the relationship between the various time gap and the SPL difference. After then, the contribution was calculated by applying OTPA to all signals in which the reference signals were compensated by the estimated time gaps. As the result, the contribution of the proposed method was almost same as the original OTPA contribution by single measurement system. This indicates that the proposed time gap compensation method worked out well to obtain correct contributions by using several measurement systems. And the method enables us to expand the application of OTPA to various products, facility, and construction using several measurement systems.

\section{Acknowledgments}

The authors thank Mueller-BBM in Germany and TOYO cooperation in Japan for supporting this work.

\section{References}

Brandl, S., Priebsch, H. -H., Brandl, F., Biermayer, W., Houldrich, R. and Sontacchi, A., Challenges and New Solutions for Transfer Path Analysis, Proceedings of International Conference on Noise and Vibration Engineering 2008 (2008), pp.1-16.

Hirao, T., Miyamoto, Y., and Yoshida, J., Extraction of High Contributing Sound of Air Handling Unit and Noise Reduction Using Transfer Path Analysis, Proceedings of 23rd International Congress on Acoustics, Germany, (2019), pp. 1263-1270.

Klerk, D. de. and Ossipov, A., Operational Transfer Path Analysis: Theory, Guidelines and Tire Noise Application, Journal of Mechanical Systems and Signal Processing, Vol.24, No.7(2010), pp.1950-1962.

Lohrmann, M., Operational Transfer Path Analysis: Comparison with Conventional Methods, Proceedings of International Congress on Sound and Vibration 15 (2008), pp.2671-2676.

Noumura, K., and Yoshida, J., Method of Transfer Path Analysis for Vehicle Interior Sound with No Excitation Experiment, FISITA2006 Proceedings F2006D183 (2006), pp.1-10.

Nozuchi, Y., Hirao, T., and Yoshida, J., Application of Operational TPA by Employing Several Measurement Systems and Accuracy Verification, Proceedings of Mechanical Engineering Congress 2020 Japan (MECJ-20), (2020), pp. 1-5. (In Japanese)

Plunt, J., Strategy for Transfer Path Analysis (TPA) applied to Vibro-Acoustic Systems at Medium and High Frequencies, Proceedings of the 23rd International Conference on Noise \& Vibration Engineering (ISMA) (1998), pp.139 -158.

Putner, J., Lohrmann, M., and Kaltenhauser, A., Operational Transfer Path Analysis Predicting Contributions to the Vehicle Interior Noise for Different Excitations from the Same Sound Source, Proceedings of Inter-Noise 2012, (2012), pp.1-12.

Yoshida, J., and Tanaka, K., Contribution Analysis of Vibration Mode Utilizing Operational TPA, Mechanical Engineering Journal, Vol.3, No.1(2016), pp.1-15.

Yoshida, J., Tanaka, K., Nakamoto, R., and Fukasawa, K., Combination Analysis of Operational TPA and CAE Technique for Obtaining High Contributing Vibration Mode, SAE Technical Paper 2017-01-1856, (2017), pp. 1-10. 